\title{
EDUCACIÓN EN ARTE: ¿OPORTUNIDAD O RETO EN TIEMPOS DE PANDEMIA?
}

Por Laura Vivianne Bermúdez Franco*
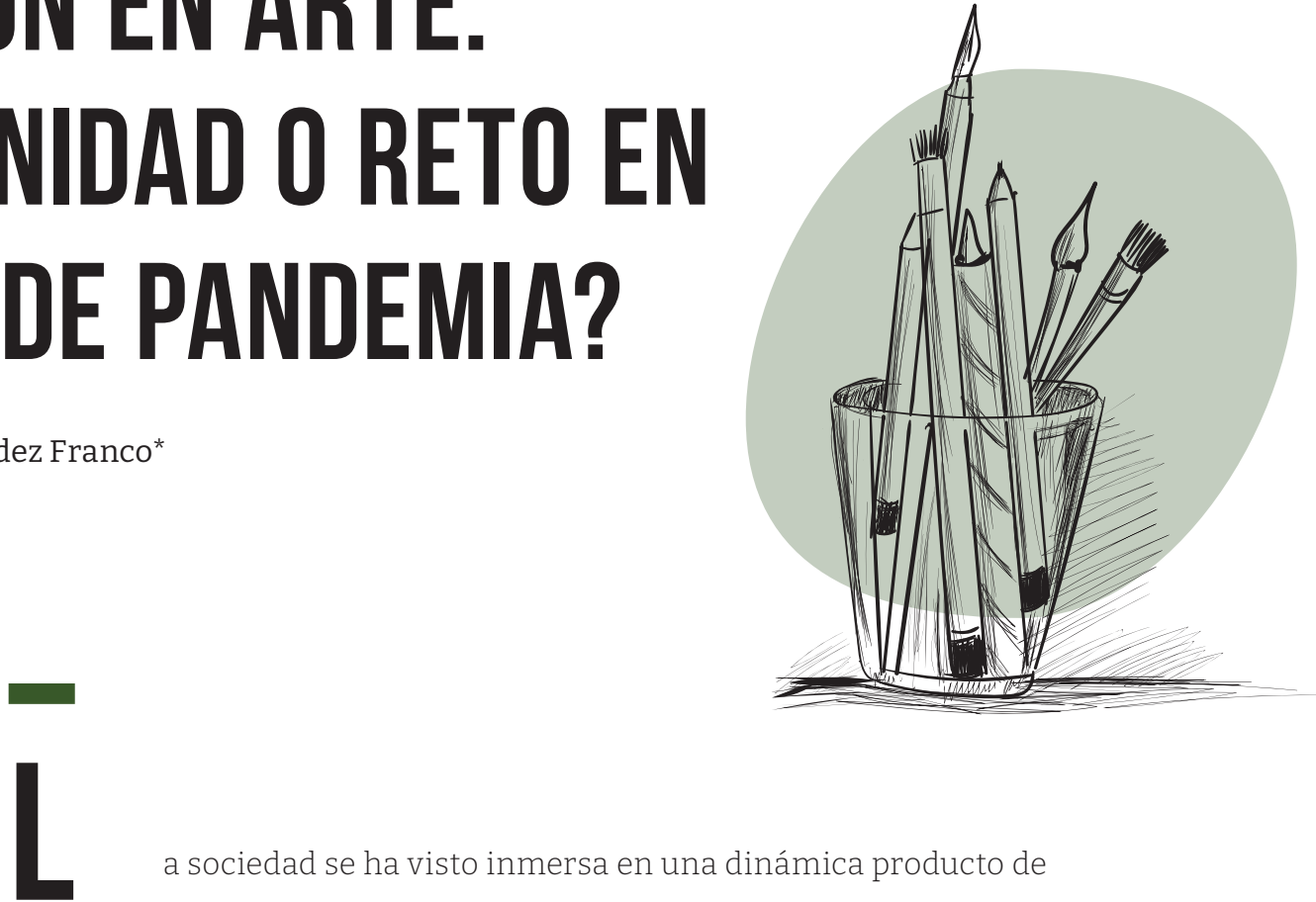

a sociedad se ha visto inmersa en una dinámica producto de lo que se ha denominado nueva normalidad, y estas dejan una serie de cuestionamientos sobre la manera en que estábamos acostumbrados a vivir. El consumo, por ejemplo, se ha orientado hacia bienes que podrían denominarse de primera necesidad y, muchos de los artículos de lujo se han quedado en eso, en ser bienes de lujo. Sin embargo, en medio de esta situación surge un debate sumamente interesante, puesto que uno de los grandes protagonistas de esta situación, además de la tecnología, ha sido el arte. La literatura, el cine, la música y el teatro se han convertido en confidentes de los dolores, angustias, miedos o incluso alegrías que toda esta situación ha generado. La palabra escrita o hablada ha sido un refugio para muchos de los que, en medio de la incertidumbre, y a veces la desesperanza, no sabemos bien qué camino se debe seguir.

En este contexto la pregunta acerca de la importancia y si se quiere llamar así, de la utilidad del arte, sale a relucir de nuevo. ¿Necesidad o lujo? Esta sería la pregunta que palpita con más y más fuerza en medio de las reflexiones producto de la pandemia, pues no solo se trata de hacer más La literatura, el cine, la música agradable la vida mientras otros buscan soluciones a los problemas que se desprenden dela situación actual, sino que se trata devolcarnuestra propiainterioridad hacia un otro que nos tiene que decir algo. En este punto, el arte trasciende el carácter de entretenimiento al que se nos ha acostumbrado y empieza a tocar

y el teatro se han convertido en

confidentes de los dolores, angustias,

miedos o incluso alegrías que toda

esta situación ha generado. 
dimensiones mucho más sustanciales en las que el diálogoy la reflexión se vuelven protagonistas. Lo anterior podría llevarnos a mencionar que el arte puede constituirse como un salvavidas en estos momentos, no por su carácter comercial, sino por la suerte de encuentro que posibilita a partir de la vinculación sensible con el mundo.

\section{En este punto, el arte trasciende el}

\section{carácter de entretenimiento al que}

\section{se nos ha acostumbrado y empieza}

\section{a tocar dimensiones mucho más}

\section{sustanciales en las que el diálogo y la}

\section{reflexión se vuelven protagonistas.}

Aunque no se deben desconocer las prácticas de consumo del arte y de la cultura, vinculadas a las nociones capitalistas, las cuales también están relacionadas con nociones principalmente económicas como el poder adquisitivo de los individuos o referencias de corte más social como los niveles de educación o estrato, lo cual se manifiesta en lo que Porro Gutiérrez (2014) ha llamado un consumo diferencial. Es importante destacar que el arte pertenece a la esencia misma del ser humano y que, desde sus comienzos, ha sentido la necesidad de representar y expresar (Muñoz, 2006) así como de comprender y reflexionar a partir del sentir. Siguiendo esta lógica, es posible comprender la razón por la que el arte ha permanecido, incluso en tiempos en que los medios virtuales son los principales canales de acceso a las obras y de comunicación con "el exterior".

Ahora bien, la relación del arte con la tecpor Ricardo Suarez, Julieth Rincón, Jhon Castro, Cesar Valbuena, Diana Pedraza, Diana Godoy y Leonardo Rivera. campos aislados, sin embargo, existen muestras de que más que contraponerse pueden entenderse como una especie de aliados. La tecnología no solo posibilita nuevas formas de expresión: nuevos sonidos, nuevos colores, sino que también hace posible una mayor difusión de las creaciones artísticas y, por qué no, de su enseñanza y aprendizaje.

Desde la mirada universitaria se gestan diferentes espacios en torno a la enseñanza y el diálogo conjunto, los cuales pretenden la exploración de las dimensiones de lo humano. Tarea nada fácil,y menos cuando pensamos que son estos medios tecnológicos los posibilitan la construcción de conocimientos. Entonces, al encontrarnos con el problema de la virtualización, o como muchos lo han llamado encuentros mediados por la tecnología en medio de la pandemia, surge la pregunta si es posible propiciar espacios de aprendizaje en torno al arte utilizando estas herramientas, que en ocasiones parecen acercarnos a los demás y otras alejarnos de lo real.

Ante este cuestionamiento, al hablar con compañeros y compartir contactos entre nosotros, tuve la oportunidad de acercarme, eso sí virtualmente, al grupo de trabajo de la Licenciatura en Artes Plásticas de la USTA; un programa que se ha constituido en la modalidad abierta y a distancia bajo la lógica de llegar a territorios donde las instituciones de educación superior normalmente no pueden llegar y, así, consolidar el proceso de formación de docentes creadores cuyas apuestas tengan cada día más y más incidencia en la transformación social. El hecho de que este programa se haya constituido bajo la modalidad señalada llama la atención, puesto que cualquiera podría preguntarse si es realmente posible enseñar Artes Plásticas por medios virtuales y cuestionar cuáles son los retos o posibilidades que se abren en este marco de actuación. Esta y otras inquietudes fue lo que nos contó el grupo de docentes ${ }^{1}$ del programa a quienes agradezco su tiempo y disposición. 


\section{Desde la mirada \\ Una década de transformaciones}

universitaria se gestan

diferentes espacios en

torno a la enseñanza

y el diálogo conjunto,

los cuales pretenden

la exploración de las

dimensiones de lo

humano.
La trayectoria de este programa, desarrollado bajo la modalidad abierta y a distancia, inicia en el año 2010. La docente Julieth Rincón, quien hoy es la coordinadora de la licenciatura, nos cuenta que este pregrado comenzó con dos profesores y ocho estudiantesy que, a pesar de contar con pocos recursos en ese momento por ser un programa nuevo, la idea era ir creciendo y así fortalecer los espacios académicos que se ofrecen. Hoy en día se cuenta con 110 estudiantes y una planta docente mucho más amplia, cuyas especialidades van desde las Artes y el Diseño Industrial hasta la Filosofía, la Historia y la Pedagogía.

El crecimiento de este programa ha estado apoyado en procesos de mejora continua y, sobre todo, en la reflexión realizada en torno a la educación a distancia, ya que, como muchos han podido experimentar en épocas de pandemia, potenciar la construcción del conocimiento por medio de herramientas virtuales no siempre es una tarea fácil, y menos cuando se piensa en la educación artística. Frente a esto, vale la pena aclarar que, si bien uno de los objetivos de la licenciatura es superar los límites que se presentan en la educación tradicional, el programacuenta con un componente presencial importante el cual se manifiesta en los talleres de dibujo, grabado o pintura que normalmente son realizados en jornadas particulares en las que los estudiantes asisten a los espacios de la Universidad para poder integrar conocimientos propios de la técnica artística.

Adicionalmente, y con elánimo de fortalecer los procesos educativos, este programa cuenta con tres semilleros académicos, cada uno con un enfoque distinto, pero fascinante para quienes escuchan y ven los resultados de su interacción. Por un lado, se encuentra el semillero Colectivo 529/62, el cual está dirigido hacia los procesos de investigación-creacióny se articula con el semillero Circuitos, curaduríay espacios expositivos, en el cual los integrantes se encargan de hacer el proceso de curaduría de las obras que se realizan en el primer semillero nombrado. Asimismo, se encuentra un tercer semillero que se constituye a partir de la colaboración entre facultades y que incluye estudiantes con condiciones diversas.

Estos grupos no se encuentran aislados de los desarrollos propios de la Facultad, ya que han sido integrados en las diferentes opciones de grado que tienen los estudiantes. A lo que se suma la posibilidad de hacerun trabajo monográfico, una pasantía, un posgrado o una ponencia internacional y la exposición de las obras que cada uno de los estudiantes realiza en espacios como el de la Casa de Bellas Artes o las salas de exposiciones de la biblioteca de la USTA.

Sin embargo, vale la pena destacar que no solamente se cuenta con espacios físicos para estas socializaciones, sino que, atendiendo primero a la modalidad a distancia que caracteriza al programa y a las condiciones propias de los estudiantes que hacen parte de ciudades como Pasto, Neiva, Bucaramanga, Amazonas, Tunja o Barranquilla, se ha potenciado eluso de espacios virtuales como Facebook ${ }^{2}$ y YouTube Y $^{3}$ para la divulgación de las obras y los procesos que se realizan en la Facultad. Espacios llenos de colores y formas que cautivan a quienes están de este lado de la pantalla.

\section{Licenciatura en artes plásticas: una apuesta por la inclusión}

Frente a la situación de contingencia que se vive a nivel nacional, frente a esta "nueva normalidad", la Facultad asume también una postura crítica producto de una mirada sensible y reflexiva sobre los acontecimientos que hoy nos 
tocan a todos. El profesor Leonardo Rivera, por ejemplo, nos cuenta que, para él desde el arte, así como desde la educación, se presentan grandes posibilidades y se abren nuevos caminos pues se reconoce que el arte es una experiencia práctica

fundamental porque es una forma de experiencia, porque es vivencial. Pero también es una forma de conocimiento que nos permite interpretary reflexionar, en el caso nuestro [de la licenciatura] desde la visualidad, pero en el arte contemporáneo desde la exploración de otros canales sensoriales como el sonido, el movimiento, laimagen ola palabra. [...] Elaporte se dajustamente porque el arte al seruna forma de conocimiento y una forma de experiencia le permite al individuo hacer ese ejercicio de introspección, pero también le permite canalizar porque también se entiende como un medio de expresión. (L. Rivera, comunicación personal, 13 de mayo de 2020)

Asimismo, el docente resalta que con los ejercicios de la licenciatura se apuesta por el enriquecimiento de la parte emocional, que permite asumirse, reconocerse, pero también encontrar nuevas formas de decir las cosas por medio del desarrollo de las capacidades y las habilidades personales y colectivas. Es por eso que asumir el proceso de formación mediante la modalidad a distancia, la cual havisto transformar los espacios

\section{REFERENCIAS}

MUÑOZ, R. (2006). Una reflexión filosófica sobre el arte. Thémata Revista de Filosofía, 36, 239-254 http://institucional. us.es/revistas/ themata/36/N4.pdf.

PORRO GUTIÉRREZ, J. (2014). Sociología del consumo cultural. Manual Atalaya. http:// atalayagestioncultural. es/capitulo/sociologia consumo-cultural de la presencialidad en encuentros sincrónicos a causa de la pandemia, implica trabajar con el potencial de autogestión de los procesos de cada uno de los miembros de la comunidad.

Frente a esto, vale la pena resaltar que muchos de los trabajos artísticos que realizan los estudiantes del programa logran vincularse con las actividades que ellos realizan dentro de las comunidades y regiones a las que pertenecen. Así, estudiantes de la licenciatura que, por ejemplo, son docentes en territorios específicos toman lo aprendido en sus clases de la USTA, lo transformany aplican en el entorno educativo en que se desenvuelven tratando de generar impactos positivos en contextos particulares. Igualmente, proyectos de carácter social como los que se desarrollan en las aulas y las comunidades sobresalen en las condiciones de pandemia, ya que permiten la creación de materiales de trabajo autónomos como parte de la práctica pedagógica que realizan los estudiantes. Se resalta así el hecho de que la licenciatura ha encontrado en la educación a distancia una oportunidad para hacer los procesos pedagógicos mucho más incluyentes, pues se generan oportunidades para que las personas puedan explorar su potencial y lo compartan con las comunidades.

Ante esto vale resaltar que la apuesta por la enseñanza mediada por herramientas tecnológicas, la cual en la licenciatura se ha venido trabajando hace casi una década: así, esta no implica una falta de rigurosidad en los procesos educativos, sino que, al contrario, requiere de una creatividad y sobre todo unos protocolos de trabajo que surgen de la experiencia cotidianay del encuentro con los miembros de la comunidad académica sin importar donde se ubiquen.

Es por esto que, ante la pregunta ¿qué podría aportarunalicenciatura comola de Artes Plásticas a situaciones como la que se vive en tiempos de pandemia?, es contundente señalar, primero, el aporte del arte en la exploracióny expresión de lo humano; segundo, a partirdelenfoquepedagógico que busca formar docentes creadores, es posible reflexionar acerca de las nuevas dinámicas de la enseñanza, de los recursos y los medios que se pueden o no usar para estos procesos y desde allí realizar apuestas personales o colectivas, que mejoren las condiciones sociales del país. Asimismo, se resalta el conocimiento en el desarrollo de los espacios sincrónicos, la sistematización de experiencias, el establecimiento de protocolos relacionados con la educación a distancia, con los que cuenta la licenciatura. Estos pueden ser de mucha ayuda para los docentes que nos vemos en la necesidad de ingresar en estos campos a causa de la situación que se vive. 